\title{
An integral LP relaxation for a drayage problem
}

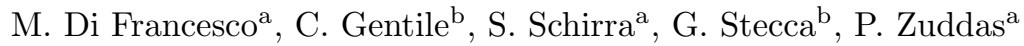 \\ ${ }^{a}$ Department of Mathematics and Computer Science, University of Cagliari \\ ${ }^{b}$ Istituto di Analisi dei Sistemi ed Informatica "Antonio Ruberti" (IASI-CNR), Rome
}

\begin{abstract}
This paper investigates a drayage problem, where a fleet of trucks must ship container loads from a port to importers and from exporters to the same port, without separating trucks and containers during customer service. We present three formulations for this problem that are valid when each truck carries one container. For the third formulation, we also assume that the arc costs are equal for all trucks, and then we prove that its continuous relaxation admits integer optimal solutions by checking that its constraint matrix is totally unimodular. Under the same hypothesis on costs, even the continuous relaxations of the first two models is proved to admit an integer optimal solution. Finally, the third model is transformed into a circulation problem, that can be solved by efficient network algorithms.
\end{abstract}

Keywords: Logistics, Vehicle Routing Problem, Drayage, Total Unimodularity

\section{Introduction}

The total unimodularity of the constraint matrix is a relevant property of optimization problems, because it can prove that the linear programming relaxation generates an optimal integral solution for the original integer programming problem. The mathematical analysis of this property was carried out in several applications (e.g. 1]), such as location and transportation problems 2, 3, as well as in possible subproblems (e.g. ship repositoning in [4]).

This paper performs this analysis in the field of the inland distribution of containers between customers and intermodal facilities, such as ports and rail 
terminals. While the vessels of liner shipping companies carry thousands of containers in maritime networks [5, 6, the supply of door-to-door services between intermodal terminals and customers must be performed by trucks carrying one or two containers. These transport activities are typically called drayage and are much more resource-intensive and energy-consuming than their maritime counterpart [7. Drayage problems were often faced by optimization methods from the Vehicle Routing Problem (VRP), in order to plan how to serve the requests of import and export customers, who must receive and ship container loads, respectively. In this paper, we focus on a drayage problem, which is motivated by a case study and can be viewed as a special variant of the VRP with Backhauls, because both pick up and delivery requests must be served, 8 , and the VRP with split delivery, because the demand of customers could be larger than the capacity of vehicles [9, 10].

To our knowledge, a mathematical analysis of integral LP relaxations has never been done in the drayage literature. The related research has focused on the proposal of algorithms aiming to solve the specific problem at hand. Two variants of drayage problems have been investigated in the literature: drop\&ipick and stay-with. In the first case, trucks are separated from or coupled with containers during customer services, thus drivers bypass packing and unpacking operations [11, 12, 13, 14, 15, 16, 17. Conversely, in stay-with operations, drivers wait for containers and trucks carry the same container(s) throughout their routes [7, 18, 19. In this paper we focus on a drayage problem with staywith operations to serve two type of transportation requests: the delivery of container loads from a port to importers and the shipment of container loads from exporters to the same port. Containers emptied at importers can be used to collect cargoes from exporters.

In most of the related studies trucks are supposed to carry one container [11, 12, 13, 14, 15, 16] and the transportation of more than one container at a time was investigated only in recent papers [17. Therefore, it is worth adopting general mathematical formulations to deal with the transportation of one or two containers. A general model for drayage problems with stay-with and multiple 
container loads per truck was proposed in [20], together with a metaheuristic solution method.

Extensive experiments with the formulation of 20] show that continuous relaxation has integer optimal solutions in the case of one-container trucks with equal costs [21]. This has been the motivation to investigate the problem under these hypotheses. In Section 2 , we present two new formulations that simplify the formulation of [20] in the case of one-container trucks. Then, in Section 3 we propose a third formulation for the case of one-container trucks with equal costs and we prove that its constraint matrix is totally unimodular and, consequently, its continuous relaxation produce integer optimal solutions. We prove that under the same hypothesis also the continuous relaxations of the first two models admits an integer optimal solution. Finally, the third formulation is transformed into a circulation problem, which can be effectively solved by very effective network flows algorithms 22 .

\section{Two formulations for one-container trucks with different costs}

In this section we describe the drayage problem we are concerned with and present two Integer Programming formulations.

We consider a port $p$, a set of importers $I=\left\{i_{1}, \ldots, i_{n}\right\}$, a set of exporters $E=\left\{l_{1}, \ldots, l_{m}\right\}$, a set of trucks $K=\left\{k_{1}, \ldots, k_{q}\right\}$. Each importer $i \in I$ requires to receive $d_{i}$ container loads. Each exporter $l \in E$ needs to ship $d_{l}$ container loads. These transportation requests must be met by using a number of trucks in $K$. Each truck starts its tour from $p$ and can visit an importer or an exporter; from an importer it can continue to an exporter or return to the port; from an exporter it must return to the port.

The problem is modeled on a graph $G(N, A)$, where $N=\{p\} \cup I \cup E$, $A=A_{1} \cup A_{2}, A_{1}=\left\{\left(j_{1}, j_{2}\right) \mid\left(j_{1}=p\right.\right.$ and $\left.j_{2} \in E\right)$ or $\left(j_{1} \in I\right.$ and $\left.j_{2} \in E\right)$ or $\left(j_{1} \in\right.$ $I$ and $\left.\left.j_{2}=p\right)\right\}, A_{2}=\left\{\left(j_{1}, j_{2}\right) \mid\left(j_{1}=p\right.\right.$ and $\left.j_{2} \in I\right)$ or $\left(j_{1} \in E\right.$ and $\left.\left.j_{2}=p\right)\right\}$. To clarify, $A_{1}$ and $A_{2}$ are the sets of arcs corresponding to the flows of empty and loaded containers, respectively. 
The model is formulated with a decision variable $x_{i j}^{k}$, which is equal to 1 if $\operatorname{arc}(i, j) \in A$ is traversed by truck $k \in K, 0$ otherwise. Moreover, $c_{i j}^{k}$ is the routing cost of truck $k \in K$ on arc $(i, j) \in A$ and $h_{p j}^{k}$ the handling cost of a container put on truck $k \in K$ at port $p$ to serve customer $j \in I \cup E$.

Fig. 1 shows a simple problem with the port $p$, one importer $i \in I$ and one exporter $l \in E$.

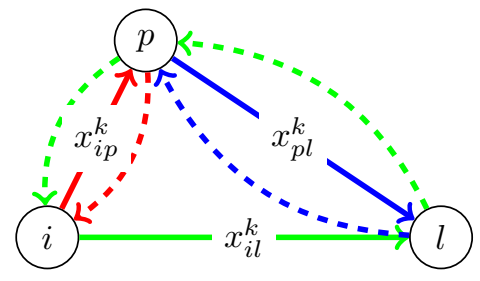

Figure 1: The three possible routes

Three possible routes can be built in the example of Fig. 1:

- from the port to an importer and from this importer to the port (red lines);

- from the port to an importer, from this importer to an exporter, from this exporter to the port (green lines);

- from the port to an exporter and from this exporter to the port (blue lines);

Dashed lines represent $\operatorname{arcs}(i, j) \in A_{2}$ traversed with loaded containers, while solid lines represent $\operatorname{arcs}(i, j) \in A_{1}$ traversed with empty containers.

The problem can be formulated as follows:

$$
\min \sum_{k \in K}\left[\sum_{j \in I \cup E}\left(c_{p j}^{k}+h_{p j}^{k}\right) x_{p j}^{k}+\sum_{i \in I ; l \in E} c_{i l}^{k} x_{i l}^{k}+\sum_{j \in I \cup E} c_{j p}^{k} x_{j p}^{k}\right]
$$




$$
\begin{array}{ll}
x_{p i}^{k}-\sum_{l \in\{p\} \cup E} x_{i l}^{k}=0 & i \in I, k \in K \\
\sum_{j \in\{p\} \cup I} x_{j l}^{k}-x_{l p}^{k}=0 & l \in E, k \in K \\
\sum_{j \in I \cup E} x_{j p}^{k}-\sum_{i \in I \cup E} x_{p i}^{k}=0 & k \in K \\
\sum_{k \in K} x_{p i}^{k}=d_{i} & i \in I \\
\sum_{k \in K} x_{l p}^{k}=d_{l} & l \in E \\
\sum_{i \in I \cup E} x_{p i}^{k} \leq 1 & k \in K \\
x_{i j}^{k} \in\{0,1\} & (i, j) \in A, k \in K
\end{array}
$$

Constraints (2), (3), and (4) are the flow conservation constraints of trucks at each importer node, at each exporter node, and at the port node, respectively; constraints (5) and (6) represent the demand constraints at each importer node and at each exporter node, respectively; constraints (7) guarantee that trucks are not used more than once.

We exploit the constraints (2) and (3) to replace variables $x_{p i}^{k}$ and $x_{l p}^{k}$ for $i \in I, l \in E, k \in K$ into constraints (4), (5), (6), (7) and into the objective function (1). In this way we obtain a new formulation, where each variable is in one-to-one correspondence with the possible routes of each vehicle. In Fig. 1 these variables are represented with solid lines. The costs associated with the removed variables (dashed lines) are transferred to the coefficients $f_{i j}^{k}$ of the other variables according to the related routes:

- $f_{i l}^{k}=\left(c_{p i}^{k}+h_{p i}^{k}\right)+c_{i l}^{k}+c_{l p}^{k}$, route $p \rightarrow i \rightarrow l \rightarrow p$, for $i \in I, l \in E, k \in K$

- $f_{i p}^{k}=\left(c_{p i}^{k}+h_{p i}^{k}\right)+c_{i p}^{k}$, route $p \rightarrow i \rightarrow p$, for $i \in I, k \in K$;

- $f_{p l}^{k}=\left(c_{p l}^{k}+h_{p l}^{k}\right)+c_{l p}^{k}$, route $p \rightarrow l \rightarrow p$, for $l \in E, k \in K$.

After the substitution of variables $x_{p i}^{k}$ and $x_{l p}^{k}$ for $i \in I, l \in E, k \in K$, constraint (4) results be an identity. Constraints (5), (6), (7) are reduced to 10), (11), 
(12), respectively, as follows:

$$
\begin{aligned}
& \min \sum_{k \in K}\left[\sum_{i \in I ; l \in E} f_{i l}^{k} x_{i l}^{k}+\sum_{i \in I} f_{i p}^{k} x_{i p}^{k}+\sum_{l \in E} f_{p l}^{k} x_{p l}^{k}\right] \\
& \sum_{k \in K}\left(x_{i p}^{k}+\sum_{l \in E} x_{i l}^{k}\right)=d_{i} \quad i \in I \\
& \sum_{k \in K}\left(x_{p l}^{k}+\sum_{j \in I} x_{j l}^{k}\right)=d_{l} \quad l \in E \\
& \sum_{i \in I} \sum_{l \in E} x_{i l}^{k}+\sum_{i \in I} x_{i p}^{k}+\sum_{i \in E} x_{p i}^{k} \leq 1 \quad k \in K \\
& x_{i j}^{k} \in\{0,1\} \quad(i, j) \in A_{1}, k \in K
\end{aligned}
$$

Constraints 10 and 11 represent demand constraints at each importer node and at each exporter node, respectively; constraints 12 guarantee that each truck is not used more than once.

The constraint matrix of the model (9)-(13) can be written with the following block structure :

$$
M=\left[\begin{array}{c|c|c|c|c}
B & B & B & \ldots & B \\
\hline D_{1} & D_{2} & D_{3} & \ldots & D_{q}
\end{array}\right],
$$

where $B \in \mathbb{R}^{(n+m) \times(n \times m+m+n)}$ and $D_{1}, \ldots, D_{q} \in \mathbb{R}^{q \times(n \times m+m+n)}$.

The block $B$ is the submatrix of the coefficients associated with constraints $(10)$, (11) and all the columns associated with truck $k_{1}$ (in Fig. 2, $B$ is represented by the rows indexed by $\left.i_{1}, \ldots, i_{n}, l_{1}, \ldots, l_{m}\right)$. The block $D_{1}$ is the submatrix of the coefficients associated with constraints $(12)$ and all the columns associated with truck $k_{1}$ (in Fig. 2, $D_{1}$ is represented by the rows indexed by $k_{1}, \ldots, k_{q}$ ). In general, the submatrix $D_{k}$ has all rows equal to zero, except the row $k$ that has all entries equal to $1, k=1, \ldots, q$.

Consider the submatrix of the constraint matrix $M$ associated with row and column indices indicated in Fig. 3 its determinant is -2 , then $M$ is not totally unimodular. 


\begin{tabular}{c|ccccccc|cccc|cccc|c}
\hline & \multicolumn{1}{|c}{$k_{1}$} & \multicolumn{1}{c}{$k_{1}$} & \multicolumn{1}{c}{$k_{1}$} \\
& $i_{1} l_{1}$ & $i_{1} l_{2}$ & $\ldots$ & $i_{2} l_{1}$ & $i_{2} l_{2}$ & $\ldots$ & $i_{n} l_{m}$ & $i_{1} p$ & $i_{2} p$ & $\ldots$ & $i_{n} p$ & $p l_{1}$ & $p l_{2}$ & $\ldots$ & $p l_{m}$ & $\ldots$ \\
\hline \hline$i_{1}$ & 1 & 1 & $\ldots$ & 0 & 0 & $\ldots$ & 0 & 1 & 0 & $\ldots$ & 0 & 0 & 0 & $\ldots$ & 0 & $\ldots$ \\
$i_{2}$ & 0 & 0 & $\ldots$ & 1 & 1 & $\ldots$ & 0 & 0 & 1 & $\ldots$ & 0 & 0 & 0 & $\ldots$ & 0 & $\ldots$ \\
$\vdots$ & & & & $\vdots$ & & & & & $\vdots$ & & & & & & $\vdots$ & $\ldots$ \\
$i_{n}$ & 0 & 0 & $\ldots$ & 0 & 0 & $\ldots$ & 1 & 0 & 0 & $\ldots$ & 1 & 0 & 0 & $\ldots$ & 0 & $\ldots$ \\
\hline \hline$l_{1}$ & 1 & 0 & $\ldots$ & 1 & 0 & $\ldots$ & $\ldots$ & 0 & 0 & $\ldots$ & 0 & 1 & 0 & $\ldots$ & 0 & $\ldots$ \\
$l_{2}$ & 0 & 1 & $\ldots$ & 0 & 1 & $\ldots$ & $\ldots$ & 0 & 0 & $\ldots$ & 0 & 0 & 1 & $\ldots$ & 0 & $\ldots$ \\
$\vdots$ & & & & $\vdots$ & & & & & $\vdots$ & & & & & & $\vdots$ & $\ldots$ \\
$l_{m}$ & 0 & 0 & $\ldots$ & 0 & 0 & $\ldots$ & 1 & 0 & 0 & $\ldots$ & 0 & 0 & 0 & $\ldots$ & 1 & $\ldots$ \\
\hline \hline$k_{1}$ & 1 & 1 & $\ldots$ & 1 & 1 & $\ldots$ & 1 & 1 & 1 & $\ldots$ & 1 & 1 & 1 & $\ldots$ & 1 & $\ldots$ \\
$k_{2}$ & 0 & 0 & $\ldots$ & 0 & 0 & $\ldots$ & 0 & 0 & 0 & $\ldots$ & 0 & 0 & 0 & $\ldots$ & 0 & $\ldots$ \\
$\vdots$ & & & & $\vdots$ & & & & & $\vdots$ & & & & & & $\vdots$ & $\ldots$ \\
$k_{q}$ & 0 & 0 & $\ldots$ & 0 & 0 & $\ldots$ & 0 & 0 & 0 & $\ldots$ & 0 & 0 & 0 & $\ldots$ & 0 & $\ldots$ \\
\hline
\end{tabular}

Figure 2: Constraint matrix $M$

\begin{tabular}{c|c|c|c|} 
& $x_{i_{1} p}^{k_{1}}$ & $x_{p l_{1}}^{k_{1}}$ & $x_{i_{1} l_{1}}^{k_{2}}$ \\
\hline$i_{1}$ & 1 & 0 & 1 \\
\hline$l_{1}$ & 0 & 1 & 1 \\
\hline$k_{1}$ & 1 & 1 & 0 \\
\hline
\end{tabular}

Figure 3: A submatrix of $M$

\section{A formulation for one-container trucks with equal costs}

If the $\operatorname{costs} c_{i j}^{k}$ and $h_{p j}^{k}$ do not depend on the choice of the truck $k$, it follows that we can define $f_{i l}=f_{i l}^{k}, f_{p i}=f_{p i}^{k}$, and $f_{l p}=f_{l p}^{k}$ for any $k \in K$. Then we can introduce a new set of integer variables:

$t_{i j}=\sum_{k \in K} x_{i j}^{k}:$ number of trucks moved along arc $(i, j) \in A_{1}$

Formulation $(9)-(13)$ can be rewritten as follows: 


$$
\begin{array}{rr}
\min _{i \in I ; l \in E} f_{i l} t_{i l}+\sum_{i \in I} f_{i p} t_{i p}+\sum_{l \in E} f_{p l} t_{p l} & \\
t_{i p}+\sum_{l \in E} t_{i l}=d_{i} & i \in I \\
t_{p l}+\sum_{i \in I} t_{i l}=d_{l} & l \in E \\
\sum_{i \in I} \sum_{l \in E} t_{i l}+\sum_{i \in I} t_{i p}+\sum_{i \in E} t_{p i} \leq|K| & \\
t_{i j} \in \mathbb{Z}_{+} & (i, j) \in A_{1}
\end{array}
$$

Equations (15) and 16 represent demand constraints at each importer node and exporter node, respectively; constraint (17) guarantees that trucks are not used more than once.

We now report a result by Ghouila-Houri [23] to prove that the constraint matrix of the model (14)-(18) is totally unimodular. Consequently, its continuous relaxation always admits an integer optimal solution.

Theorem 1. Let $A \in \mathbb{Z}^{m \times n}$. A is totally unimodular if and only if for every $C \subseteq\{1, \ldots, m\}$, there exists a partition of $C$ into $C_{1}$ and $C_{2}$ such that

$$
\left|\sum_{c_{1} \in C_{1}} a_{c_{1} j}-\sum_{c_{2} \in C_{2}} a_{c_{2} j}\right| \leq 1 \quad \forall j=1, \ldots, n .
$$

Theorem 2. The constraint matrix of formulation (14)-(18) is totally unimodular.

Proof. Let us denote with $A$ the constraint matrix of (14)-(18). This matrix is depicted in Fig. 2 as the restriction of matrix $M$ limited to rows indexed by $I \cup$ $E \cup\left\{k_{1}\right\}$, where $I=\left\{i_{1}, \ldots, i_{n}\right\}$ and $E=\left\{l_{1}, \ldots, l_{m}\right\}$, and the columns indexed by set $X_{1} \cup X_{2} \cup X_{3}$, where $X_{1}=\left\{\left(i, l, k_{1}\right) \mid i \in I, l \in E\right\}, X_{2}=\left\{\left(i, p, k_{1}\right) \mid i \in\right.$ $I\}, X_{3}=\left\{\left(p, l, k_{1}\right) \mid l \in E\right\}$. Let us denote with $I^{\prime}$ and $E^{\prime}$ any subset of $I$ and $E$, respectively. We will use Theorem 1 to prove that $A$ is totally unimodular. We distinguish two cases. 
- Case 1: Let $C$ be a subset of rows of $A$ such that $k_{1} \in C$. Then we can write $C=I^{\prime} \cup E^{\prime} \cup\left\{k_{1}\right\}$ and choose the partition: $C_{1}=I^{\prime} \cup E^{\prime}, C_{2}=\left\{k_{1}\right\}$. We indicate in the following table the sum of the elements of each column:

\begin{tabular}{|c|c|c|c|c|} 
& & $X_{1}$ & $X_{2}$ & $X_{3}$ \\
\hline \hline & $\sum_{c_{1} \in I^{\prime}} a_{c_{1} j}$ & $1 \vee 0$ & $1 \vee 0$ & 0 \\
\hline+ & $\sum_{c_{1} \in E^{\prime}} a_{c_{1} j}$ & $1 \vee 0$ & 0 & $1 \vee 0$ \\
\hline- & $\sum_{c_{2} \in C_{2}} a_{c_{2} j}$ & 1 & 1 & 1 \\
\hline \hline$=$ & & $1 \vee 0 \vee-1$ & $0 \vee-1$ & $0 \vee-1$ \\
\hline
\end{tabular}

For every column indexed by $(i, l, k) \in X_{1} \cup X_{2} \cup X_{3}$ the sum of the elements in $I^{\prime} \cup E^{\prime}$ may be 2,1 , or 0 . Subtracting the row indexed by $k_{1}$, we obtain $1,0,-1$. Then condition 19 holds and Theorem 1 applies.

- Case 2: Let $C$ be a subset of rows of $A$ such that $k_{1} \notin C$. Then we can write $C=I^{\prime} \cup E^{\prime}$ and choose the partition: $C_{1}=I^{\prime}, C_{2}=E^{\prime}$.

\begin{tabular}{|c|c|c|c|c|} 
& & $X_{1}$ & $X_{2}$ & $X_{3}$ \\
\hline \hline & $\sum_{c_{1} \in C_{1}} a_{c_{1} j}$ & $1 \vee 0$ & $1 \vee 0$ & 0 \\
\hline- & $\sum_{c_{2} \in C_{2}} a_{c_{2} j}$ & $1 \vee 0$ & 0 & $1 \vee 0$ \\
\hline \hline$=$ & & $1 \vee 0 \vee-1$ & $0 \vee-1$ & $0 \vee-1$ \\
\hline
\end{tabular}

For every column indexed by $(i, l, k) \in X_{1} \cup X_{2} \cup X_{3}$ the sum of the elements in $I^{\prime}$ may be 1 or 0 . Subtracting the row indexed by $E^{\prime}$, we obtain $1,0,-1$. Then condition $(19)$ holds and Theorem 1 applies.

Summing up Case 1 and Case 2, we can conclude that $A$ is totally unimodular, and that formulation (14)-18 has a continuous relaxation that always admits an integer optimal solution.

Model (14)-18) can be efficiently solved by min cost flow techniques (see, e.g., [22]) by transforming it into a circulation problem over a network $\tilde{G}(\tilde{N}, \tilde{A})$ defined as follows (Fig. 4 shows a simple example with a port $p$, one importer $i \in I$ and one exporter $l \in E)$ :

- $\tilde{N}$ contains two nodes for each node $u \in N$, say $u^{s}$ and $u^{e}$; 
- $\tilde{A}$ contains $\operatorname{arcs}\left(u^{s}, v^{e}\right)$ for each $\operatorname{arc}(u, v) \in A$, and $\operatorname{arcs}\left(u^{e}, u^{s}\right)$ for each node $u \in N$;

- associate costs $f_{u v}$ on the $\operatorname{arcs}\left(u^{s}, v^{e}\right)$ for each $(u, v) \in A_{1}$, and zero otherwise;

- associate lower and upper bounds equal to $d_{u}$ for each $\operatorname{arc}\left(u^{e}, u^{s}\right)$ and $u \in I \cup E$; associate upper bound equal to $K$ to the arc $\left(p^{e}, p^{s}\right)$ for the port $p$.

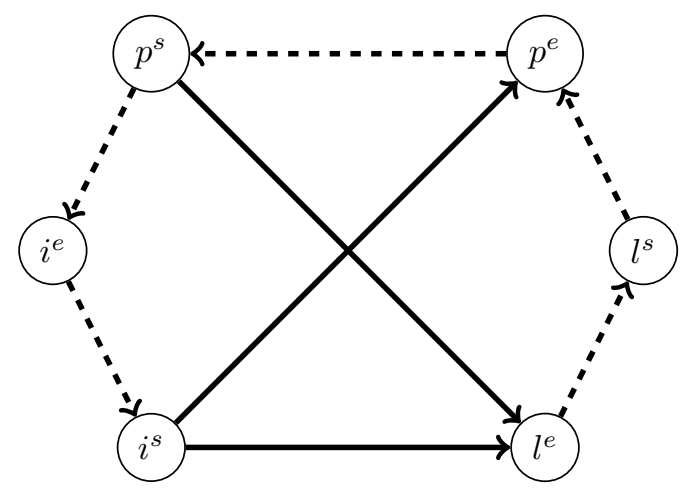

Figure 4: The network $\tilde{G}$

Denoting with $y$ the vector of flows over the network $\tilde{G}$, the LP formulation 
of the circulation problem can be summarized as follows:

$$
\begin{array}{lr}
\min _{i \in I ; l \in E} f_{i l} y_{i^{s} l^{e}}+\sum_{i \in I} f_{i p} y_{i^{s} p^{e}}+\sum_{l \in E} f_{p l} y_{p^{s} l^{e}} & \\
y_{i^{e} i^{s}}=y_{i^{s} p^{e}}+\sum_{l \in E} y_{i^{s} l^{e}} & i \in I \\
y_{p^{s} i^{e}}=y_{i^{e} i^{s}} & i \in I \\
y_{l^{e} l^{s}}=y_{p^{s} l^{e}}+\sum_{i \in I} y_{i^{s} l^{e}} & l \in E \\
y_{l^{s} p^{e}}=y_{l^{e} l^{s}} & \\
y_{p^{e} p^{s}}=\sum_{i \in I} y_{i^{s} p^{e}}+\sum_{l \in E} y_{l^{s} p^{e}} & \\
y_{p^{e} p^{s}}=\sum_{i \in I} y_{p^{s} i^{e}}+\sum_{l \in E} y_{p^{s} l^{e}} & \\
y_{i^{e} i^{s}}=d_{i} & \\
y_{l^{e} l^{s}}=d_{l} & \\
y_{p^{e} p^{s} \leq} \leq \mid K & \\
y_{u v} \in \mathbb{Z}_{+} & (u \in E \\
&
\end{array}
$$

Theorem 3. The circulation problem defined on the network $\tilde{G}$ and model (14)(18) are equivalent.

Proof. It sufficient to check that each solution of the circulation problem on $\tilde{G}$ can be mapped into a solution of model (14)-18 with equal objective function values and viceversa.

Let $t$ be a solution of model (14)-18 and define $y_{u^{s} v^{e}}=t_{u v}$ for each arc $(u, v) \in A_{1}$. Then $y_{p^{s} i^{e}}=y_{i^{e} i^{s}}=\sum_{v \in E \cup\{p\}} t_{i v}$ for each importer $i \in I$, because of constraints 21) and 222; $y_{l^{s} p^{e}}=y_{l^{e} l^{s}}=\sum_{v \in I \cup\{p\}} t_{v l}$ for each exporter $l \in E$, because of constraints 223 and 24. For constraint 25 $y_{p^{e} p^{s}}=\sum_{i \in I} t_{i p}+$ $\sum_{l \in E} \sum_{v \in I \cup\{p\}} t_{v l}=\sum_{i \in I} t_{i p}+\sum_{i \in I ; l \in E} t_{i l}+\sum_{l \in E} t_{p l}$. Then constraint 26) is satisfied because its right-hand-side correspond to the above value for $y_{p^{e}} p^{s}$, indeed $\sum_{i \in I} y_{p^{s} i^{e}}+\sum_{l \in E} y_{p^{s} l^{e}}=\sum_{i \in I} \sum_{v \in E \cup\{p\}} t_{i v}+\sum_{l \in E} t_{p l}=\sum_{i \in I} t_{i p}+$ $\sum_{i \in I ; l \in E} t_{i l}+\sum_{l \in E} t_{p l}=y_{p^{e} p^{s}}$. Finally, constraints 27), 28), and 29) are 
satisfied, by previous computations, their left-hand-sides correspond to the lefthand-sides of constraints (15), (16), and (17), respectively. Then $y$ is a feasible circulation for (21)-(30). The values of the objective function (14) for solution $t$ and of the objective function 20 for solution $y$ are equal by construction.

Now, we prove the opposite. Let $y$ be a solution for the circulation problem. Then define $t_{u v}=y_{u^{s} v^{e}}$ for each $(u, v) \in A_{1}$. Constraints 15) are satisfied thanks to 21] and (27); constraints (16) are satisfied thanks to 23) and 28); constraint (17) is satisfied because of constraints (25), 224), (23), and (29). As before, the objective functions values of $y$ and $t$ are the same by construction.

Finally, we can prove that also the continuous relaxations of formulation (11)-(8) and of formulation (9)-(13) admit an integer optimal solution when the arc costs are equal for all vehicles.

Proposition 1. If the costs $c_{i j}^{k}$ and $h_{p j}^{k}$ do not depend on $k$, then the continuous relaxation of formulation (9)-13) admits an integer optimal solution.

Proof. By Theorem 2 formulation (14)-(18) admits an integer optimal solution $t^{*}$ for its relaxation. Associated with $t^{*}$ there exists a dual optimal solution $\left(\lambda^{*}, \mu^{*}\right)$ with $\lambda^{*} \in \mathbb{R}^{|I|+|E|}$ and $\mu^{*} \in \mathbb{R}$ such that

$$
\begin{array}{rr}
\sum_{i \in I ; l \in E} f_{i l} t_{i l}^{*}+\sum_{i \in I} f_{i p} t_{i p}^{*}+\sum_{l \in E} f_{p l} t_{p l}^{*}=\sum_{i \in I} d_{i} \lambda_{i}^{*}+\sum_{l \in E} d_{l} \lambda_{l}^{*}+|K| \mu^{*}, \\
f_{i l}-\lambda_{i}^{*}-\lambda_{l}^{*}-\mu^{*} \geq 0 & i \in I, l \in E, \\
f_{i p}-\lambda_{i}^{*}-\mu^{*} \geq 0 & i \in I, \\
f_{p l}-\lambda_{l}^{*}-\mu^{*} \geq 0 & l \in E,
\end{array}
$$

where condition (31) states that primal objective function value of $14-(18)$ is equal to its dual objective function value, and (32)-34 are the dual constraints for variables $t_{i l}, t_{i p}, t_{p l}, i \in I, l \in E$, respectively.

Then we can define a solution $\tilde{x}$ and a corresponding dual solution for (9)(13) as follows: 
- for each variable $t_{i l}^{*}>0\left(t_{i p}^{*}>0, t_{p l}^{*}>0\right)$ define a set of vehicles $K_{i l}$ $\left(K_{i p}, K_{p l}\right.$, respectively) such that $\left|K_{i l}\right|=t_{i l}^{*}\left(\left|K_{i p}\right|=t_{i p}^{*},\left|K_{p l}\right|=t_{p l}^{*}\right.$, respectively); each vehicle $k \in K$ belongs to at most one set in the family $\mathcal{F}_{K}=\left\{K_{i l} \mid i \in I, l \in E\right\} \cup\left\{K_{i p} \mid i \in I\right\} \cup\left\{K_{p l} \mid l \in E\right\} ;$

- set $\tilde{x}_{i l}^{k}=1$ for $k \in K_{i l}, \tilde{x}_{i p}^{k}=1$ for $k \in K_{i p}, \tilde{x}_{p l}^{k}=1$ for $k \in K_{p l}$, and $\tilde{x}_{i l}^{k}=0, \tilde{x}_{i p}^{k}=0, \tilde{x}_{p l}^{k}=0$, otherwise;

- define the dual solution for the relaxation of $(9)-(13)$ as $(\tilde{\lambda}, \tilde{\mu}) \in \mathbb{R}^{|I|+|E|+|K|}$ such that $\tilde{\lambda}=\lambda^{*}$ and $\tilde{\mu}^{k}=\mu^{*}$ for each $k \in K$.

We check that $(\tilde{x}, \tilde{\lambda}, \tilde{\mu})$ satisfies primal and dual constraints for $9-913$ and attains the same objective function value for the relaxation of $(9)-13$ and for its dual, respectively. Primal feasibility for 10 and 11 is straightforward because $\sum_{k \in K} \tilde{x}_{i l}^{k}=t_{i l}^{*}, \sum_{k \in K} \tilde{x}_{i p}^{k}=t_{i p}^{*}, \sum_{k \in K} \tilde{x}_{p l}^{k}=t_{p l}^{*}$; primal feasibility for 12 comes from the condition that the sets $K_{i l}, K_{i p}, K_{p l}$ are pairwise disjoint for all $i \in I, l \in E$, so at most one variable in 12 takes value 1 . The dual constraints of the continuous relaxation (9)- $(13)$ are the following:

$$
\begin{array}{rr}
f_{i l}^{k}-\tilde{\lambda}_{i}-\tilde{\lambda}_{l}-\tilde{\mu}^{k} \geq 0 & i \in I, l \in E, k \in K, \\
f_{i p}^{k}-\tilde{\lambda}_{i}-\tilde{\mu}^{k} \geq 0 & i \in I, k \in K, \\
f_{p l}^{k}-\tilde{\lambda}_{l}-\tilde{\mu}^{k} \geq 0 & l \in E, k \in K .
\end{array}
$$

Dual feasibility of $(\tilde{\lambda}, \tilde{\mu})$ comes from definition of $\tilde{\lambda}$ and $\tilde{\mu}^{k}$ and from conditions (32)-(34). Finally, we check that with respect to reformulation (9)-(13) the dual objective value for $(\tilde{\lambda}, \tilde{\mu})$ is equal to its primal objective value for $\tilde{x}$ :

$$
\begin{aligned}
& \sum_{k \in K} \tilde{\mu}^{k}+\sum_{i \in I} \tilde{\lambda}_{i} d_{i}+\sum_{l \in E} \tilde{\lambda}_{l} d_{l}= \\
= & \mu^{*}|K|+\sum_{i \in I} \lambda_{i}^{*} d_{i}+\sum_{l \in E} \lambda_{l}^{*} d_{l}= \\
= & \sum_{i \in I ; l \in E} f_{i l} t_{i l}^{*}+\sum_{i \in I} f_{i p} t_{i p}^{*}+\sum_{l \in E} f_{p l} t_{p l}^{*}= \\
= & \sum_{i \in I ; l \in E} f_{i l} \sum_{k \in K} \tilde{x}_{i l}^{k}+\sum_{i \in I} f_{i p} \sum_{k \in K} \tilde{x}_{i p}^{k}+\sum_{l \in E} f_{p l} \sum_{k \in K} \tilde{x}_{p l}^{k},
\end{aligned}
$$

and as consequence $\tilde{x}$ and $(\tilde{\lambda}, \tilde{\mu})$ are a pair of primal-dual optimal solutions for the relaxation of $(9)-13)$ and $\tilde{x}$ is integer by construction. 
An easy consequence of Proposition 1 is that also the continuous relaxation of reformulation (1)-(8) admits an integer optimal solution when the arc costs do not depend on the vehicle. Indeed, it is sufficient to first determine the solution for (9)-(13) and then set the variables $x_{p i}^{k}$ and $x_{l p}^{k}$ by using constraints (2) and (3). This can be summarized in the following corollary:

Corollary 1. If the costs $c_{i j}^{k}$ and $h_{p j}^{k}$ do not depend on $k$, then the continuous relaxation of reformulation (1)-(8) admits an integer optimal solution.

\section{Conclusion}

We proposed three new formulations for a drayage problem with stay-with policy in the case of trucks carrying one container. More precisely, we simplified the model in 20. providing two new formulations that can handle different arc costs for trucks. We showed that the constraint matrices of these formulations are not totally unimodular. Then we proposed a third formulation valid if the arc costs do not depend on the vehicle and we proved that its constraint matrix is totally unimodular. As a consequence, the continuous relaxation of this formulation always produce integer optimal solutions. In addition, we also proved that, under the same hypothesis, also the first two formulations admit an integer optimal solution for the respective continuous relaxations.

Finally, we showed that the third model can be used to define a circulation problem over a network that can be solved by efficient algorithms. The impact of these results is their possible use in decomposition methods to solve more complex problems, for which the problem at hand is a subproblem. For example, it is of interest to solve the original drayage problem in [20] with variables associated with trucks with either one or two containers by means of decomposition methods such as Benders' decomposition. Indeed, this problem can be represented as a model where the vector of variables associated with trucks carrying 1 or 2 containers are represented with vectors $w^{1}$ and $w^{2}$ respectively, and where the Benders' decomposition can be applied by considering $w^{1}$ as the vector of "easy variables". The minimization subproblem associated with variables 
$w^{1}$ is equivalent to the third model, and it can be efficiently used in the Benders' decomposition by exploiting the transformation into the circulation problem as above mentioned. That enables us to take advantage of the fact that in realworld instances more than $90 \%$ of the trucks carry only one container. In a future work we will compare the solution of the general drayage problem by the original formulation in [20] with the Benders' decomposition approach.

\section{Acknowledgements}

Massimo Di Francesco, Giuseppe Stecca and Paola Zuddas have been partially supported by the PRIN program of the Ministry of Instruction University and Research (MIUR) by the project "Smart PORt Terminals" - SPORT, code 2015XAPRKF. Claudio Gentile has been partially supported by the project PRIN 2015B5F27W 005 "Nonlinear and Combinatorial Aspects of Complex Networks". The authors are also gratefully to the Sardinia Regional Government for the financial support of the PhD program of Silvia Shirra (P.O.R. Sardegna F.S.E. Operational Programme of the Autonomous Region of Sardinia, European Social Fund 2007-2013 - Axis IV Human Resources, Objective 1.3, Line of Activity 1.3.1.).

\section{References}

[1] A. Charnes, M. Kress, Two simple applications of the unimodularity property, Operations Research Letters 14 (14) (1993) 257-260.

[2] A. Tamir, Totally balanced and totally unimodular matrices defined by center location problems, Discrete Applied Mathematics 3 (16) (1987) 245263.

[3] P. Wei, Y. Cao, D. Sun, Total unimodularity and decomposition method for large-scale air traffic cell transmission model, Transportation Research Part B: Methodological 53 (2013) 1-16. 
[4] S. Wang, Essential elements in tactical planning models for container liner shipping, Transportation Research Part B: Methodological 54 (2013) 84-99.

[5] S. Wang, Q. Meng, Z. Liu, Containership scheduling with transit-timesensitive container shipment demand, Transportation Research Part B: Methodological 54 (2013) 68-83.

[6] S. Wang, Q. Meng, C.-Y. Lee, Liner container assignment model with transit-time-sensitive container shipment demand and its applications, Transportation Research Part B: Methodological 90 (2016) 135-155.

[7] L. B. Reinhardt, D. Pisinger, S. Spoorendonk, M. M. Sigurd, Optimization of the drayage problem using exact methods, INFOR: Information Systems and Operational Research 54 (1) (2016) 33-51.

[8] P. Toth, D. Vigo, VRP with backhauls, in: P. Toth, D. Vigo (Eds.), The Vehicle Routing Problem, SIAM Monographs on Discrete Mathematics and Applications, Philadelphia, PA, 2002, pp. $195-224$.

[9] M. Dror, G. Laporte, P. Trudeau, Vehicle routing with split deliveries, Discrete Applied Mathematics 50 (1994) 239-254.

[10] C. Archetti, M. G. Speranza, A. Hertz, A tabu search algorithm for the split delivery vehicle routing problem, Transportation Science 40 (1) (2006) 64-73.

[11] X. Wang, A. C. Regan, Local truckload pickup and delivery with hard time window constraints, Transportation Research Part B: Methodological 36 (2002) 97-112.

[12] H. Jula, M. Dessouky, P. Ioannou, A. Chassiakos, Container movement by trucks in metropolitan networks: modeling and optimization, Transportation Research Part E: Logistics and Transportation Review 41 (3) (2005) 235-259. 
[13] R. Zhang, W. Yun, H. Kopfer, Heuristic-based truck scheduling for inland container transportation, OR Spectrum 32 (3) (2010) 787-808.

[14] R. Zhang, W. Yun, I. Moon, Modeling and optimization of a container drayage problem with resource constraints, International journal of Production Economics 133 (1) (2011) 351-359.

[15] K. Braekers, A. Caris, G. Janssens, Integrated planning of loaded and empty container movements, OR Spectrum 35 (2) (2013) 457-478.

[16] J. Nossack, E. Pesch, A truck scheduling problem arising in intermodal container transportation, European Journal of Operational Research 230 (3) (2013) 666-680.

[17] M. Vidovic, D. Popovic, B. Ratkovic, G. Radivojevic, Generalized mixed integer and VNS heuristic approach to solving the multisize containers drayage problem, International Transactions in Operational Research 24 (3) (2017) 583-614.

[18] A. Imai, E. Nishimura, J. Current, A lagrangian relaxation-based heuristic for the vehicle routing with full container load, European journal of Operational Research 176 (1) (2007) 87-105.

[19] A. Caris, G. Janssens, A local search heuristic for the pre- and end-haulage of intermodal container terminals, Computers and Operations Research 36 (10) (2009) 2763-2772.

[20] M. Lai, T. G. Crainic, M. Di Francesco, P. Zuddas, An heuristic search for the routing of heterogeneous trucks with single and double container loads, Transportation Research Part E: Logistics and Transportation Review 56 (2013) 108-118.

[21] S. Schirra, Algebraic structural analysis of a vehicle routing problem of heterogeneous trucks. identification of the properties allowing an exact approach., Ph.D. thesis, University of Cagliari (2017). 
[22] A. Goldberg, An efficient implementation of a scaling minimum-cost flow algorithm, J. Algorithms 22 (1997) 1-29.

[23] A. Ghouila-Houri, Caractérisation des matrices totalement unimodulaires, Comptes Rendus Hebdomadaires des Scéances de l'Académie des Sciences (Paris) 254 (1962) 1192-1194. 